\title{
A EVOLUÇÃO DAS DESIGUALDADES POR CATEGORIAS DE ESCOLARIDADE ENTRE 1996 E 2004: UMA ANÁLISE COM REGRESSÕES QUANTÍLICAS*
}

\author{
$\operatorname{Marcos} \operatorname{Roch}^{* *}$ \\ Maria de Fátima Sales de Souza Campos ${ }^{* *}$
}

Maurício Vaz Lobo Bittencourt ${ }^{* * *}$

RESUMO Este trabalho investiga se existem modificações relevantes entre os quantis de rendimento nos retornos por escolaridade, ou seja, se os retornos por escolaridade mudaram ao longo do período para quantis diferentes, e entre estratos educacionais para os diversos quantis de rendimento (se os rendimentos relativos por quantil mudaram entre indivíduos com anos de escolaridade diversa). Foi feita análise diferenciada da evolução dessas categorias através da metodologia das regressões quantílicas nas equações de salário para um continuum de tempo, de 1996 a 2004, usando a base de dados da Pesquisa Nacional por Amostra de Domicílios (Pnad). O cenário geral dos resultados mostra que a análise quantílica por rendimento acentua a convergência entre os quantis de renda e estratos educacionais, assim como um padrão de comportamento de relativa constância para os quantis de renda mais altos. A análise vista por estratos educacionais enfatiza a queda relativa dos rendimentos do ensino fundamental e a relativa constância dos rendimentos do estrato da pós-graduação.

* Artigo recebido em 25 de junho de 2007 e aprovado em 5 de novembro de 2009.

** Professor assistente na Pontifícia Universidade Católica de São Paulo (PUC-SP) e doutorando pela Fundação Getúlio Vargas, e EESP-FGV, e-mail: marcosrocha@gvmail.br

*** Professora adjunta da Universidade Estadual de Londrina (UEL), e-mail: mfscampos@uol.com.br

**** Professor adjunto e Coordenador do Programa de Pós-Graduação em Desenvolvimento Econômico da Universidade Federal do Paraná (PPGDE-UFPR), e-mail: mbittencourt@ufpr.br 
Palavras-chave: mercado de trabalho; diferenciais de salário; regressões quantílicas

Código JEL: A20; C81; I21; J31

\title{
THE EVOLUTION OF WAGE INEQUALITIES IN URBAN BRAZIL \\ BY SCHOOLING LEVELS FOR THE PERIOD 1996 TO 2004: \\ A QUANTILE REGRESSION APPROACH
}

\begin{abstract}
This study investigates if there are relevant modifications between income quantiles in returns to schooling, that is, if the schooling returns in the different income quantiles has changed, and between schooling categories in the different income quantiles (if the relative income quantile has changed in the different schooling categories). The study of these schooling categories was performed through the use of the quantile regressions approach in the wage equation for the period 1996-2004, using data base drawn from Pesquisa Nacional por Amostra de Domicílios (Pnad). The big picture from the results has shown that the quantile analysis by income underlines the convergence between the income quantiles and schooling categories and also a relative steady pattern for the higher quantile income individuals. The analysis made by schooling categories underlines the relative loss of income for the elementary school level and the relative steady behavior for the graduate studies level. The joint results stresses that the higher quantile income and schooling categories follows a different path than that of the others, by showing less changes or relative losses over the period in analysis.
\end{abstract}

Key words: labor market; wage differentials; quantile regression 


\section{INTRODUÇÃO}

Durante a década de 1990, o Brasil experimentou profundas mudanças estruturais, tais como o processo de abertura comercial no início dessa década, a privatização de setores importantes na economia (a exemplo dos setores elétrico e de telecomunicações), a liberalização dos fluxos de capitais e um programa de estabilização alicerçado em uma âncora cambial, o Plano Real, com reflexos importantes sobre toda a economia e, em especial, sobre o mercado de trabalho.

Reformas econômicas no início dos anos 1990 se estenderam a muitos países em desenvolvimento: diversos artigos documentam substancial mudança nos salários e empregos nesse trânsito (Paul e Robinson, 1997; Revenga, 1997; Mitner e Wright, 1998; Arbache, 1999). As reformas econômicas que começaram no final dos anos 1980 e se aprofundaram nos anos 1990, no Brasil, tiveram impacto significativo no mercado de trabalho. Salm, Saboia e Carvalho (1997) apontam para grandes aumentos de produtividade no setor manufatureiro desde 1990. Barros et al. (1996) e Moreira e Najberg (1997) encontraram redução no emprego industrial como resultado da abertura comercial.

Não só as reformas econômicas afetam o mercado de trabalho e motivam sua análise. O Brasil é um dos países com maiores níveis de desigualdade de renda do mundo. Uma parte considerável dessa desigualdade está relacionada à desigualdade de rendimentos do trabalho entre os indivíduos ocupados. Barros et al. (1996) mostram que cerca de 60\% das desigualdades de renda familiar per capita poderiam ser eliminadas, se a remuneração dos indivíduos ocupados fosse a mesma. De acordo com Ramos (2007), aproximadamente $40 \%$ das desigualdades no Brasil podem ser explicados pela heterogeneidade dos indivíduos no mercado de trabalho, das quais $80 \%$ podem ser atribuídos à educação. A compreensão de como os rendimentos do trabalho são determinados e de como a educação torna-se um elemento explicativo para os diferenciais salariais torna-se relevante, em função do índice de desigualdade observado para a economia brasileira. ${ }^{1}$

Dada a importância da educação formal para explicar rendimentos e desigualdades, assunto intensamente discutido em diversos trabalhos e para diversas funções, o objetivo deste trabalho é investigar se existem modificações 
relevantes entre os quantis de rendimento nos retornos por escolaridade (ou seja, se os retornos por escolaridade mudaram ao longo do período para quantis diferentes) e entre estratos educacionais para os diversos quantis de rendimento (se os rendimentos relativos por quantil mudaram entre indivíduos com anos de escolaridade diversos). Para isso, foram utilizadas regressões quantílicas e variáveis categóricas para cada estrato educacional. O foco é entender se o rendimento dos indivíduos (por quantis) afeta os retornos dos trabalhadores urbanos em salário por anos de escolaridade. Foi construída uma série de variáveis categóricas para cada um dos estratos de educação formal (ensino fundamental, ensino médio etc.) com vistas à análise diferenciada da evolução dessas categorias; os resultados tiveram interpretação gráfica. O trabalho utiliza a metodologia das regressões quantílicas nas equações de salário para um continuum de tempo, de 1996 a 2004, usando a base de dados da Pesquisa Nacional por Amostra de Domicílios (Pnad). ${ }^{2}$

O uso da metodologia quantílica estende a análise convencional dos diferenciais de salário, contribuindo para a observação das mudanças que ocorrem dentro da estrutura da distribuição dos rendimentos. Diversos papers que estudam o assunto, como Buchinsky (1994 e 1995), Chamberlain (1994), Fitzenberger e Kurz (1997), Silveira Neto e Campelo (2003) e Machado e Mata (2005) sugerem o uso de regressões quantílicas como forma de avaliar as modificações na distribuição condicional da renda. Buchinsky (1994 e 1995) é responsável por diversos trabalhos seminais no tratamento por quantis das equações salário. Em uma extensiva análise do assunto, seus resultados mostram que os retornos à escolaridade e experiência apresentaram padrões semelhantes, embora a magnitude dos coeficientes tenha diferido ao longo dos quantis da distribuição salarial, com retornos maiores à escolaridade e experiência para os indivíduos que se situam no topo da distribuição de salários.

O artigo está estruturado da forma que se segue: além desta introdução, a seção seguinte detalha alguns aspectos teóricos do arcabouço que deu origem à equação salarial minceriana, qual seja, a teoria do capital humano e suas implicações. A seção 2 apresenta a metodologia de regressões quantílicas. O modelo econométrico utilizado e a base de dados se encontram na seção 3. A seção 4 traz os resultados das regressões, sendo que a seção 5 conclui com uma apreciação geral do trabalho. 


\section{FUNDAMENTAÇÃO TEÓRICA}

A teoria econômica tem modelos teóricos diversos para descrever a forma com que o mercado de trabalho aloca os rendimentos entre os trabalhadores. Pode-se dizer que a primeira explicação da determinação dos salários amplamente aceita no mainstream veio com o modelo neoclássico original, que dizia que os rendimentos eram derivados da ação da oferta e demanda por mão de obra. No equilíbrio, os trabalhadores deveriam ser remunerados de acordo com sua produtividade marginal na produção dos bens e serviços oferecidos na economia. Com o tempo, o modelo se mostrou insuficiente para explicar o funcionamento do mercado, em especial no que se refere à incapacidade em explicitar como os investimentos em educação poderiam determinar diferenças salariais. Tornava-se necessário um modelo de escolhas racionais que explicasse os diferenciais de salários no mercado de trabalho, incorporando no esquema o comportamento dos indivíduos em seu investimento em educação. É isso que a teoria do capital humano se propôs fazer.

A teoria do capital humano veio ampliar o enfoque da teoria neoclássica inicial, ao defender que a não homogeneidade do fator trabalho era devida a diversos determinantes importantes na formação da mão de obra, em especial a escolaridade. ${ }^{3}$ Mais tarde, a variável experiência foi também introduzida como elemento importante de determinação dos diferenciais salariais. Nesse contexto, a demanda pela educação está relacionada positivamente com os aumentos nos ganhos vitalícios ou com os benefícios cotidianos que uma formação escolar proporciona.

Os ganhos médios dos trabalhadores devem elevar-se com o aumento do nível de educação. Se isso não acontecesse, os incentivos para os estudantes investirem em educação - o que, na maioria das vezes, é muito dispendioso em tempo e dinheiro - desapareceriam. Além disso, é empiricamente constatado que o perfil idade/rendimentos dos trabalhadores tem uma forma convexa, o que a teoria do capital humano explica em termos da evolução do treinamento no emprego. Os ganhos são baixos a princípio, enquanto o indivíduo investe em treinamento, mas irão elevar-se, e de forma rápida, à medida que vão sendo adquiridas novas qualificações que proporcionam remunerações adicionais. Portanto, o aumento mais rápido nos ganhos irá ocorrer no início da carreira do trabalhador, depois, tende a reduzir sua 
taxa, e, finalmente, irá cair. É essa dinâmica da evolução da variável idade/ ganhos que reproduzirá uma forma convexa à função.

Mincer (1974) argumenta que o indivíduo dedica-se à educação no começo de sua carreira, quando seu estoque de capital humano é baixo. Essa fase é dedicada pelo indivíduo apenas ao aprendizado, até o período em que este escolherá entrar no mercado de trabalho, quando passa a combinar o processo de aprendizado com algum trabalho que executa. Uma função-salário minceriana básica capta o perfil salário-idade na seguinte forma, por exemplo:

$$
\text { In } y=\beta_{0}+\beta_{1} s+\beta_{2} s^{2}+\beta_{3} x+\beta_{4} x^{2}+\mu
$$

onde:

yé a taxa de salário do trabalhador;

$s$ é o número de anos de estudo;

$s^{2}$ é o número de anos de estudo ao quadrado;

$x$ é o número de anos de experiência no mercado de trabalho;

$x^{2}$ é o termo experiência ao quadrado;

$\mu$ é um termo erro aleatório.

O termo $\beta_{1}$ é o coeficiente de escolaridade, que mensura a taxa de retorno marginal à educação. $\mathrm{O}$ coeficiente $\beta_{3}$ mensura o impacto de um ano adicional de experiência sobre os rendimentos. A experiência é modelada como faz Mincer (1974), que usa a idade de cada trabalhador para construir uma variável proxy em que é assumido que os trabalhadores entram na força de trabalho logo após completarem sua educação, e que $s+6$ é a idade de conclusão da formação educacional. ${ }^{4} \mathrm{O}$ coeficiente $\beta_{4}$, designando a experiência ao quadrado, capta com o termo quadrático os retornos decrescentes de salário ao aumento marginal dos anos de escolaridade.

Seja $x$ a experiência, de acordo com a especificação convencional minceriana, tem-se que:

$$
x=\text { idade }-s-6
$$

Os rendimentos decrescentes obtidos por cada ano de escolaridade adicional são captados na equação de salários pelo coeficiente do termo quadrático da educação $\left(s^{2}\right)$, cujo sinal espera-se ser negativo na estimação. Para 
a variável experiência ao quadrado $\left(x^{2}\right)$, espera-se que seu sinal seja negativo, como forma de refletir a existência dos rendimentos decrescentes; ou seja, o sinal negativo sinaliza que, para níveis maiores de experiência, haverá um retorno proporcionalmente inferior da taxa de rendimentos. ${ }^{5}$

\section{REGRESSÕES QUANTÍLICAS}

A técnica semiparamétrica de regressões quantílicas tem despertado a atenção de numerosos pesquisadores e servido de instrumental para diversas constatações empíricas, ajudando a esclarecer como essas modificações alteram a estrutura dentro da distribuição.

As regressões quantílicas foram introduzidas na literatura especializada por Koenker e Basset (1978). De acordo com Buchinsky (1998) e Yu et al. (2003), os modelos quantílicos têm várias características úteis:

(a) podem ser usados para caracterizar a distribuição condicional inteira de uma variável dependente, dado um conjunto de variáveis explicativas;

(b) têm uma representação de programação linear que torna as estimações fáceis;

(c) a função objetivo da regressão quantílica é uma soma ponderada dos desvios absolutos;

(d) analisam toda a distribuição, enquanto a abordagem de MQO convencional só analisa a média da relação entre as variáveis ("efeito causal médio");

(e) as diferentes soluções nos distintos quantis podem ser interpretadas como diferenças na resposta da variável dependente a mudanças nos regressores ao longo dos vários pontos da distribuição condicional da variável dependente.

Seguindo a metodologia proposta inicialmente por Koenker e Basset (1978), assume-se que $y_{i},(i=1, \ldots, n)$, é uma amostra de observações do logaritmo dos rendimentos, e que $X_{i}$ é um vetor $K \times 1$ que é constituído por variáveis como educação, experiência, e diversas outras variáveis de controle. O modelo de regressão quantílica pode, então, ser definido como se segue:

$$
y_{i}=X_{i}^{\prime} \beta_{\theta}+u_{\theta i}
$$




$$
\mathrm{Q}_{\theta}\left(y_{i}: X_{i}\right)=X_{i}^{\prime} \beta_{\theta}, \theta \in(0,1)
$$

onde $\mathrm{Q}_{\theta}\left(y_{i}: X_{i}\right)$ representa o quantil do log do salário horário condicional dado o vetor de regressores. A regressão quantílica $\theta$ pode ser definida como a solução do problema seguinte:

$$
\min _{\beta} \frac{1}{n}\left[\sum_{i: y_{i} \geq x_{i}^{\prime} \beta} \theta\left|y_{i}-x_{i}^{\prime} \beta_{\theta}\right|+\sum_{i: y_{i} \leq x_{i}^{\prime} \beta}(1-\theta)\left|y_{i}-x_{i}^{\prime} \beta_{\theta}\right|\right]=\min _{\beta} \frac{1}{n} \sum_{i=1}^{n} \rho_{\theta}\left(u_{\theta i}\right)
$$

onde $\rho_{\theta}$ (.) é conhecido como função check, que, por sua vez, é definida como:

$$
\rho_{\theta}(.)=\left\{\begin{array}{l}
\theta u_{\theta i} \text { se } u_{\theta i} \geq 0 \\
(\theta-1) u_{\theta i} \text { se } u_{\theta i}<0
\end{array}\right.
$$

A interpretação dos coeficientes das regressões quantílicas é conceitualmente análoga à de regressões MQO. No caso deste último, os coeficientes das regressões medem a influência das variáveis exógenas sobre a média condicional da variável dependente, enquanto na regressão quantílica os coeficientes representam a influência dos regressores sobre a variável dependente condicional ao $\theta$-ésimo quantil.

Em um artigo que examina a evolução dos retornos para a educação em Portugal durante os anos 1980 e começo de 1990, Hartog, Pereira e Vieira (2001) mostram que a modelagem na média (MQO) perde diversas características importantes da estrutura salarial, uma vez que os efeitos da educação sobre os salários podem não ser constantes ao longo da distribuição, como mostram os trabalhos de Buchinsky (1994 e 1995). Os autores constataram que a desigualdade salarial em Portugal aumentou no período, e os retornos à educação tiveram papel importante nesse processo.

No Brasil, Silveira Neto e Campelo (2003), utilizando a técnica de regressões quantílicas, concluíram que as maiores disparidades de renda observadas entre as regiões Nordeste e Sudeste ocorrem nos quantis inferiores da distribuição condicional da renda, o que não foi observado por outros autores que utilizaram regressões MQO.

Menezes Filho, Fernandes e Picchetti (2006) analisaram a desigualdade salarial no Brasil ao longo da distribuição condicional, concluindo que o 
aumento no nível de escolaridade da força de trabalho nas décadas de 1980 e 1990 não reduziu a desigualdade de rendimentos, embora haja uma perspectiva de reversão nos próximos anos.

Justo (2007) observou que os retornos à educação no Brasil entre $1992 \mathrm{e}$ 2002 são mais elevados para os indivíduos que se situam no topo da distribuição de salários. No entanto, os salários dos indivíduos das faixas inferiores da distribuição de salários têm crescido mais fortemente, reduzindo os diferenciais salariais. Uma possível explicação, segundo o autor, seria o maior investimento em educação por parte dos trabalhadores que se situam nos limites inferiores da distribuição salarial.

Martins e Pereira (2004) utilizaram regressões quantílicas estimando retornos à educação para entender a relação entre escolaridade e desigualdade salarial. As evidências para trabalhadores masculinos de 16 países para meados dos anos 1990 sugerem um fato robusto: os retornos para a escolaridade são maiores para indivíduos mais qualificados, condicionais às características observáveis. $\mathrm{O}$ resultado sugere que a escolaridade tem um impacto positivo sobre os diversos níveis de desigualdade salarial. Os autores apontam fatores como over-education e interações de treinamento e habilidade e qualidade escolar para explicá-lo.

\section{MODELO ECONOMÉTRICO E BASE DE DADOS}

A base de dados utilizada foi a Pesquisa Nacional por Amostra de Domicílios (Pnad), para os anos 1996 a 2004, exceto 2000, quando a pesquisa não foi realizada. Para a análise empírica, o peso do indivíduo na amostra da Pnad foi considerado como fator de ponderação. ${ }^{6}$ Foram selecionados apenas os indivíduos economicamente ativos e retirados os trabalhadores agrícolas: a amostra se restringe a trabalhadores urbanos. A diferença substancial de funcionamento dos mercados de trabalhos agrícola e urbano sugere o uso dessa distinção. Ademais, a metodologia da Pnad mudou: apenas a partir de 2002 os indivíduos de área rural da região Norte foram incorporados na amostra. Foram também excluídos os aposentados e pensionistas de instituto de previdência federal, estadual ou municipal. A tabela 1 apresenta as estatísticas descritivas das variáveis utilizadas nas regressões. 
Tabela 1: Estatística

\begin{tabular}{|c|c|c|c|c|c|c|c|c|}
\hline & \multicolumn{2}{|c|}{1996} & \multicolumn{2}{|c|}{1997} & \multicolumn{2}{|c|}{1998} & \multicolumn{2}{|c|}{1999} \\
\hline & Média & $\begin{array}{l}\text { Desvio- } \\
\text { padrão }\end{array}$ & Média & $\begin{array}{l}\text { Desvio- } \\
\text { padrão }\end{array}$ & Média & $\begin{array}{l}\text { Desvio- } \\
\text { padrão }\end{array}$ & Média & $\begin{array}{l}\text { Desvio- } \\
\text { padrão }\end{array}$ \\
\hline $\ln w$ & 0,570 & 0,948 & 0,609 & 0,943 & 0,633 & 0,929 & 0,639 & 1,003 \\
\hline Brancos-dummy & 0,544 & 0,498 & 0,537 & 0,499 & 0,532 & 0,499 & 0,533 & 0,496 \\
\hline Casados-dummy & 0,773 & 0,419 & 0,769 & 0,422 & 0,765 & 0,424 & 0,760 & 0,743 \\
\hline Experiência & 19.319 & 12.320 & 19.382 & 12.318 & 19.439 & 12.364 & 19.488 & 19.356 \\
\hline Experiência ${ }^{2}$ & 524.996 & 594.250 & 527.400 & 596.794 & 530.719 & 595.145 & 535.200 & 535.827 \\
\hline Formais-dummy & 0,524 & 0,499 & 0,519 & 0,500 & 0,514 & 0,500 & 0,505 & 0,510 \\
\hline $\begin{array}{l}\text { Fundamental } \\
\text { I-dummy }\end{array}$ & 0,254 & 0,435 & 0,254 & 0,435 & 0,243 & 0,429 & 0,233 & 0,176 \\
\hline $\begin{array}{l}\text { Fundamental } \\
\text { II-dummy }\end{array}$ & 0,309 & 0,462 & 0,303 & 0,460 & 0,301 & 0,459 & 0,299 & 0,270 \\
\hline Homens-dummy & 0,579 & 0,494 & 0,581 & 0,493 & 0,579 & 0,494 & 0,572 & 0,555 \\
\hline Médio-dummy & 0,242 & 0,428 & 0,248 & 0,432 & 0,263 & 0,440 & 0,277 & 0,352 \\
\hline Nordeste-dummy & 0,261 & 0,439 & 0,263 & 0,440 & 0,270 & 0,444 & 0,272 & 0,269 \\
\hline Norte-dummy & 0,075 & 0,263 & 0,076 & 0,266 & 0,076 & 0,265 & 0,074 & 0,123 \\
\hline $\begin{array}{l}\text { Pós-Graduação- } \\
\text { dummy }\end{array}$ & 0,075 & 0,263 & 0,077 & 0,267 & 0,077 & 0,266 & 0,079 & 0,089 \\
\hline $\begin{array}{l}\text { Recebimento de } \\
\text { Renda-dummy }\end{array}$ & 0,057 & 0,231 & 0,053 & 0,225 & 0,056 & 0,229 & 0,055 & 0,097 \\
\hline $\begin{array}{l}\text { Chefe de Família- } \\
\text { dummy }\end{array}$ & 0,449 & 0,497 & 0,451 & 0,498 & 0,454 & 0,498 & 0,454 & 0,450 \\
\hline $\begin{array}{l}\text { Centro-Oeste- } \\
\text { dummy }\end{array}$ & 0,108 & 0,310 & 0,113 & 0,316 & 0,116 & 0,320 & 0,114 & 0,119 \\
\hline Escolaridade & 8.112 & 4.209 & 8.163 & 4.230 & 8.275 & 4.217 & 8.430 & 9.211 \\
\hline Sudeste & 0,374 & 0,484 & 0,369 & 0,482 & 0,358 & 0,479 & 0,360 & 0,319 \\
\hline Sul & 0,183 & 0,386 & 0,179 & 0,384 & 0,180 & 0,384 & 0,180 & 0,170 \\
\hline Superior-dummy & 0,043 & 0,202 & 0,043 & 0,203 & 0,044 & 0,204 & 0,044 & 0,057 \\
\hline $\begin{array}{l}\text { Escolaridade versus } \\
\text { Experiência } \\
\end{array}$ & 132.757 & 98.662 & 133.853 & 99.143 & 136.104 & 100.427 & 138.913 & 151.979 \\
\hline Whw & 3.016 & 5.728 & 3.124 & 7.186 & 3.149 & 5.840 & 3.135 & 4.461 \\
\hline Observações & & 447 & & 377 & & 570 & 101 & 792 \\
\hline
\end{tabular}

Fonte: Resultados da pesquisa a partir das Pnads. 
descritiva das variáveis

\begin{tabular}{|c|c|c|c|c|c|c|c|}
\hline \multicolumn{2}{|c|}{2001} & \multicolumn{2}{|c|}{2002} & \multicolumn{2}{|c|}{2003} & \multicolumn{2}{|c|}{2004} \\
\hline Média & $\begin{array}{l}\text { Desvio- } \\
\text { padrão }\end{array}$ & Média & $\begin{array}{l}\text { Desvio- } \\
\text { padrão }\end{array}$ & Média & $\begin{array}{l}\text { Desvio- } \\
\text { padrão }\end{array}$ & Média & $\begin{array}{l}\text { Desvio- } \\
\text { padrão }\end{array}$ \\
\hline 0,890 & 0,923 & 0,751 & 0,915 & 0,829 & 0,910 & 1,003 & 0,890 \\
\hline 0,500 & 0,499 & 0,518 & 0,500 & 0,513 & 0,500 & 0,496 & 0,500 \\
\hline 0,437 & 0,427 & 0,756 & 0,430 & 0,750 & 0,433 & 0,743 & 0,437 \\
\hline 12.696 & 12.466 & 19.394 & 12.513 & 19.353 & 12.595 & 19.356 & 12.696 \\
\hline 599.894 & 600.124 & 532.683 & 595.888 & 533.180 & 598.900 & 535.827 & 599.894 \\
\hline 0,500 & 0,500 & 0,502 & 0,500 & 0,500 & 0,500 & 0,510 & 0,500 \\
\hline 0,381 & 0,422 & 0,209 & 0,406 & 0,199 & 0,399 & 0,176 & 0,381 \\
\hline 0,444 & 0,458 & 0,288 & 0,453 & 0,284 & 0,451 & 0,270 & 0,444 \\
\hline 0,497 & 0,495 & 0,569 & 0,495 & 0,564 & 0,496 & 0,555 & 0,497 \\
\hline 0,477 & 0,448 & 0,306 & 0,461 & 0,321 & 0,467 & 0,352 & 0,477 \\
\hline 0,443 & 0,445 & 0,270 & 0,444 & 0,271 & 0,444 & 0,269 & 0,443 \\
\hline 0,329 & 0,261 & 0,111 & 0,314 & 0,113 & 0,316 & 0,123 & 0,329 \\
\hline 0,285 & 0,270 & 0,080 & 0,271 & 0,083 & 0,276 & 0,089 & 0,285 \\
\hline 0,297 & 0,227 & 0,059 & 0,236 & 0,074 & 0,262 & 0,097 & 0,297 \\
\hline 0,498 & 0,498 & 0,459 & 0,498 & 0,450 & 0,498 & 0,450 & 0,498 \\
\hline 0,324 & 0,318 & 0,119 & 0,323 & 0,117 & 0,321 & 0,119 & 0,324 \\
\hline 4.127 & 4.196 & 8.682 & 4.197 & 8.887 & 4.161 & 9.211 & 4.127 \\
\hline 0,466 & 0,480 & 0,330 & 0,470 & 0,331 & 0,470 & 0,319 & 0,466 \\
\hline 0,375 & 0,384 & 0,171 & 0,376 & 0,169 & 0,375 & 0,170 & 0,375 \\
\hline 0,232 & 0,205 & 0,050 & 0,217 & 0,053 & 0,224 & 0,057 & 0,232 \\
\hline 111.261 & 101.758 & 142.561 & 105.728 & 146.069 & 107.485 & 151.979 & 111.261 \\
\hline 13.650 & 5.322 & 3.537 & 7.434 & 3.764 & 6.551 & 4.461 & 13.650 \\
\hline \multicolumn{2}{|c|}{125791} & \multicolumn{2}{|c|}{114113} & \multicolumn{2}{|c|}{118189} & \multicolumn{2}{|c|}{125791} \\
\hline
\end{tabular}


A especificação das equações-salário estimadas neste artigo é a seguinte:

$$
\begin{aligned}
& \ln w=\alpha+\beta_{0} X_{1}+\beta_{1} X_{2}+\beta_{2} X_{3}+\beta_{3} X_{4}+\beta_{4} X_{5}+\beta_{5} X_{6}+ \\
& \beta_{6} X_{6}^{2}+\beta_{7} X_{7}+\beta_{8} D_{1}+\beta_{9} D_{2}+\beta_{10} D_{3}+\beta_{11} D_{4}+\beta_{12} D_{5}+ \\
& \beta_{13} D_{6}+\beta_{14} D_{7}+\beta_{15} D_{8}+\beta_{16} D_{9}+\varepsilon
\end{aligned}
$$

onde:

- $X_{1}$ é uma variável categórica com valor 1 se o indivíduo tem anos de escolaridade completo ou incompleto no ensino fundamental I, e 0 se não;

- $X_{2}$ é uma variável categórica com valor 1 se o indivíduo tem anos de escolaridade completo ou incompleto no ensino fundamental II, e 0 se não;

- $X_{3}$ é uma variável categórica com valor 1 se o indivíduo tem anos de escolaridade completo ou incompleto no ensino médio, e 0 se não;

- $X_{4}$ é uma variável categórica com valor 1 se o indivíduo tem anos de escolaridade completo ou incompleto no ensino médio, e 0 se não;

- $X_{5}$ é uma variável categórica com valor 1 se o indivíduo tem anos de escolaridade completo ou incompleto na pós-graduação, e 0 se não;

- $X_{6}$ é uma proxy da experiência do indivíduo;

- $X_{6}^{2}$ é o quadrado da proxy de experiência do indivíduo;

- $X_{7}$ é o termo do produto dos anos de escolaridade e experiência do indivíduo;

- $D_{1}$ é uma variável categórica com valor 1 se o indivíduo é do sexo masculino, e 0 se não;

- $D_{2}$ é uma variável categórica com valor 1 se o indivíduo é da cor branca, e 0 se não;

- $D_{3}$ é uma variável categórica com valor 1 se o indivíduo é casado, e 0 se não;

- $D_{4}$ é uma variável categórica com valor 1 se o indivíduo trabalha no setor formal com carteira assinada, e 0 se não;

- $D_{5}$ é uma variável categórica com valor 1 se o indivíduo é o responsável pela família, e 0 se não;

- $D_{6}$ é uma variável categórica com valor 1 se o indivíduo vive na região Centro-Oeste do país, e 0 se não; 
- $D_{7}$ é uma variável categórica com valor 1 se o indivíduo vive na região Nordeste do país, e 0 se não;

- $D_{8}$ é uma variável categórica com valor 1 se o indivíduo vive na região Sul do país, e 0 se não;

- $D_{9}$ é uma variável categórica com valor 1 se o indivíduo vive na região Centro-Oeste do país, e 0 se não;

- $\varepsilon$ é o termo erro estocástico da regressão.

A variável $\ln w$ é o logaritmo neperiano do salário por horas semanais. É uma variável obtida do trabalho principal do indivíduo da amostra obtida da Pnad. Detalhes a respeito da amostra final obtida através dos filtros, como as médias e desvios-padrão de cada variável, além do número de indivíduos em cada ano, são encontrados na tabela1.

\section{RESULTADOS E DISCUSSÃo}

Nesta seção são analisados os resultados advindos das equações salariais quantílicas. Todas as equações tiveram uma formatação gráfica dos coeficientes estimados ao longo dos anos cobertos pela análise para facilitar a interpretação. Os coeficientes de todas as variáveis das regressões foram significativos no nível de significância de $5 \%$. O R ${ }^{2}$ das equações MQO convencionais situa-se na faixa de 0,45 , como é comum obter-se na literatura de equações-salário - uma vez que foi desconsiderada, na análise, a filiação industrial de cada trabalhador, o que reduz o potencial de explicação das equações, mas simplifica a exposição com uma especificação mais enxuta. A ênfase deste trabalho recai, portanto, na análise das mudanças ao longo do tempo dos parâmetros das variáveis de escolaridade, mais do que no comportamento de outros fatores que possivelmente expliquem os diferenciais de salário.

Cada variável de escolaridade agrega indivíduos com o nível de escolaridade completo ou incompleto de um estrato: se o indivíduo tem o ensino superior incompleto ou completo como anos de escolaridade, a variável "Ensino Superior" assume o valor igual a 1; caso contrário, assume valor igual a 0 . Foram criadas dummies para cada um dos estratos de escolaridade, tendo como grupo-base os indivíduos sem escolaridade formal. A razão da incorporação das dummies está no interesse em como as posições relativas dos estratos escolares evoluiu, mais do que a análise do retorno por escolaridade de um ano adicional propriamente dito. ${ }^{7}$ 
A estratégia utilizada é a análise comparativa das variáveis, categóricas ou não, e seus resultados ao longo do tempo, do quantil, ou de alguma categoria da amostra de interesse (estrato educacional, por exemplo). Na análise gráfica e por tabelas, as variáveis categóricas foram devidamente corrigidas para informar o impacto percentual sobre o salário que descrevem, ou seja, os valores das variáveis dummies para o logit quantílico foram ajustados por meio da relação:

valor percentual $=[\exp ($ coeficiente da variável $)-1]$

Para o ano 2000, quando não foi realizada a pesquisa da Pnad, a análise se fez com a média dos coeficientes dos anos imediatamente anterior e subsequente. Os gráficos foram construídos a partir das dummies das regressões quantílicas, que informam, condicional a cada quantil, o diferencial percentual de salários para os atributos da variável categórica, tudo o mais mantido constante. Salienta-se que os quantis foram ordenados dos menores para os maiores rendimentos: assim, o quantil $q=25$ se localiza na parte inferior da distribuição (menores rendimentos), enquanto o quantil $q=90$ situa-se na parte superior da distribuição de salários (em que se concentram indivíduos com maiores rendimentos).

As regressões que deram origem aos gráficos mostram resultados para as variáveis de controle que já são esperados para as cross-sections: a educação afeta positivamente os salários, assim como a experiência, para todos os períodos e quantis. Os coeficientes da variável experiência têm sinal positivo e suas estimativas variam de 0,024 a 0,038, sendo todas significativas. O termo de interação de escolaridade e experiência mostrou-se nulo ou fracamente positivo, porém significativo. A experiência ao quadrado, apesar de ser significativa estatisticamente, foi positiva e próxima de zero na maioria das regressões, sugerindo quase nenhuma influência dos rendimentos decrescentes da experiência sobre o rendimento dos trabalhadores. Esse resultado pode sugerir que, uma vez controlados efeitos individuais além daqueles da equação minceriana tradicional, a experiência deixa de ser tão importante para explicar diferenciais de salário.

Outros resultados mostram que, ceteris paribus, homens ganham mais do que mulheres, assim como brancos mais do que não brancos, trabalhadores formais mais do que informais, chefes de família mais 
com relação a outros membros da residência, como é comumente constatado na literatura.

Pode-se visualizar, nos quadros do gráfico 1, um resultado que é compartilhado por todos os quantis da distribuição: a convergência dos retornos percentuais de escolaridade entre os estratos; ou seja, fica evidenciada uma redução da desigualdade entre diplomas, controladas as características observáveis. Entretanto, menor convergência se dá no quantil q = 90: o estrato de pós-graduação mantém o coeficiente de relação aproximadamente igual a 6 com relação ao grupo de indivíduos sem escolaridade. ${ }^{8}$ De modo geral, o estrato de pós-graduação mantém, ceteris paribus, quase o dobro em termo de retornos salariais com relação ao estrato de ensino superior.

As maiores discrepâncias reveladas pela separação entre os quantis estão entre os grupos de escolaridade ao longo dos quantis. No quantil $q=25, o$ extremo superior do estrato educacional analisado, a pós-graduação, tem seus retornos à escolaridade mantidos mais ou menos constantes ao longo do período, com coeficiente de relação de 3,5 no tocante ao grupo de indivíduos sem escolaridade. Os indivíduos com ensino superior mantêm um coeficiente de relação de cerca de 1,5. A partir do quantil q = 50, a distinção entre grupos superiores e inferiores de diplomas se acentua: no quantil $q=50$, a relação entre o rendimento por escolaridade controlada para indivíduos de pós-graduação atinge uma média de coeficiente de relação a indivíduos sem escolaridade de aproximadamente 5; para $\mathrm{q}=75$, esse estrato chega a atingir coeficiente de relação de aproximadamente 6 no intervalo 1998-1999, havendo ligeira redução até 2004.

Para os outros estratos inferiores à pós-graduação, o retorno à escolaridade relativa entre os estratos é mais uniforme durante o período de análise. Para todos os quantis, o ensino superior mantém uma média de coeficiente de relação 3 .

No gráfico 2, os resultados para cada estrato são avaliados separadamente: o foco é sobre o comportamento dentro dos quantis. Todos os estratos por educação para a maioria dos quantis da distribuição experimentaram queda relativa de seus rendimentos (exceto para pós-graduação em q = 90). Os estratos fundamental I, fundamental II, ensino médio e superior experimentaram quedas menos bruscas. Observa-se, ainda, uma tendência à convergência para todos os quantis, exceto para a pós-graduação. O grande 


\section{Grafico 1: Evolução dos rendimentos por estrato educacional ao longo de 1996-2004, para os quantis 25, 50, 75 e 90}
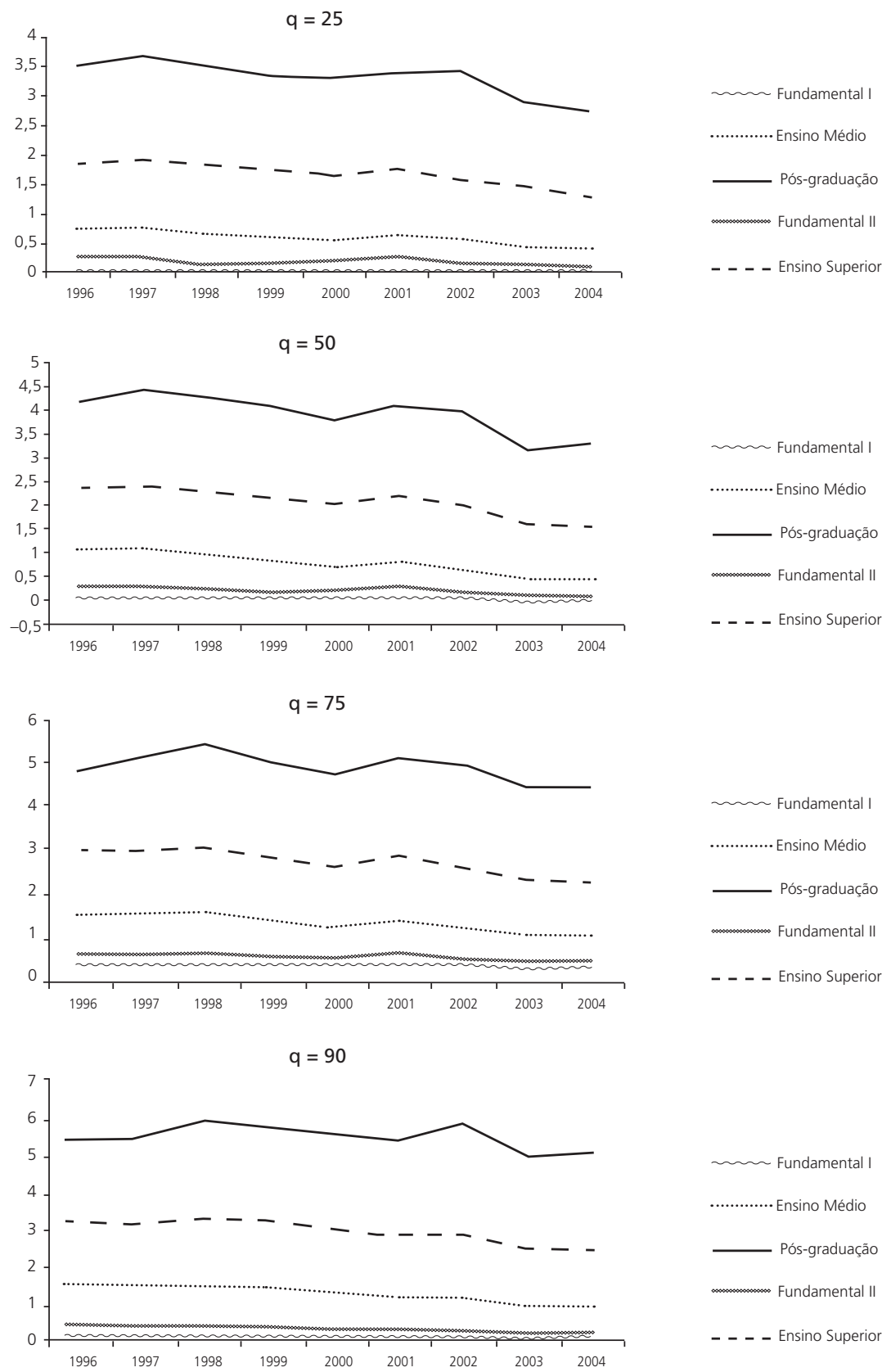

Fonte: Construção a partir das variáveis categóricas das regressões. Todas as variáveis têm significância inferior a 5\% 
Gráfico 2: Evolução dos rendimentos para os

quantis 25, 50, 75 e 90 por estrato educacional

Fundamental I

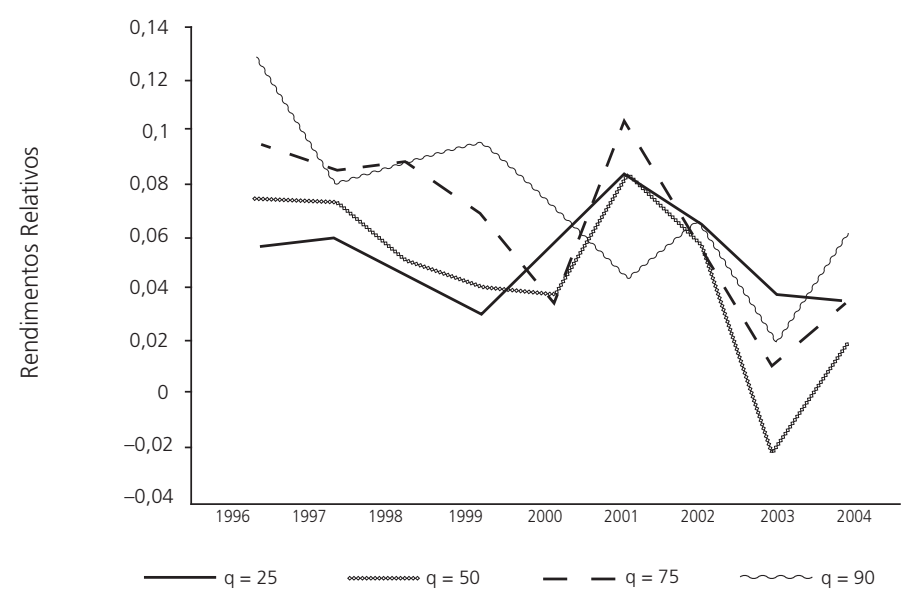

Fundamental II

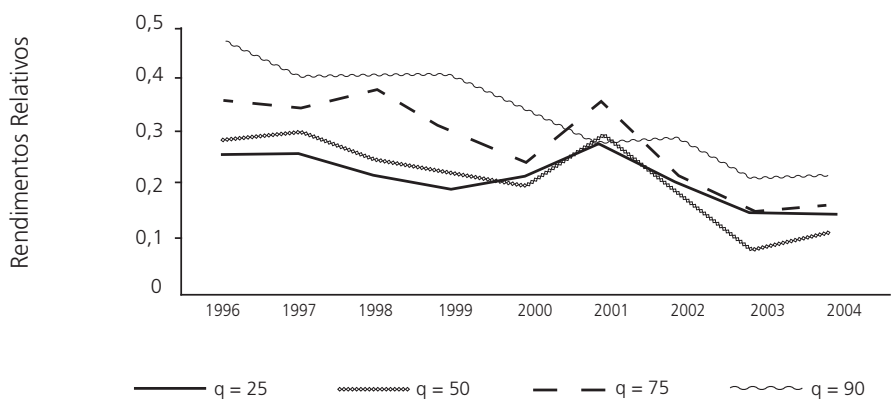

Ensino Médio

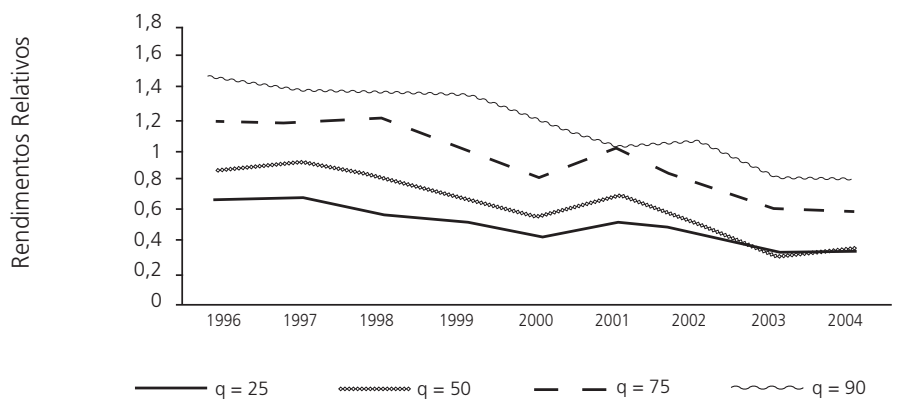




\section{Continuação do gráfico 2: Evolução dos rendimentos para os quantis 25, 50, 75 e 90 por estrato educacional}

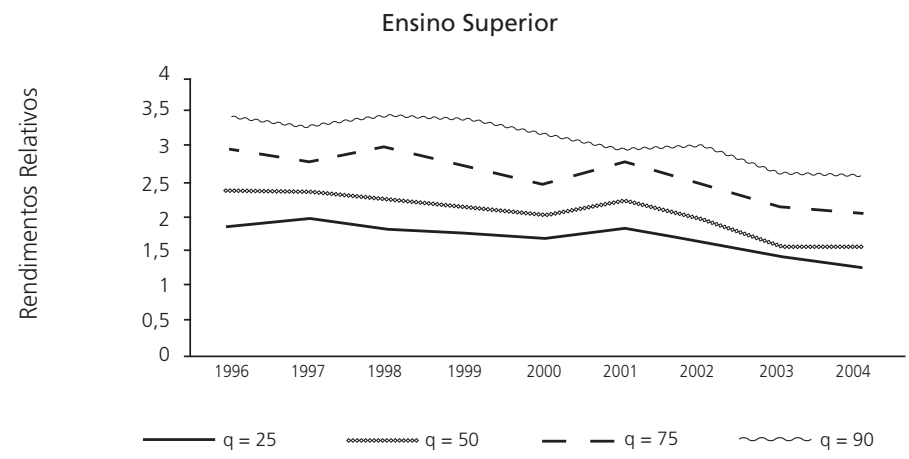

Pós-graduação

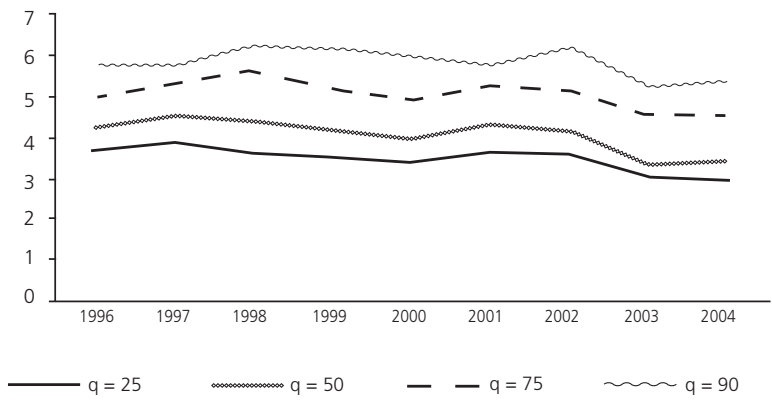

Fonte: Construção a partir das variáveis categóricas das regressões. Todas as variáveis têm significância inferior a 5\%

destaque é o comportamento para as curvas para a pós-graduação, que se mantiveram relativamente estáveis durante todo o período. Pode-se dizer que as curvas determinaram convergência para todos os estratos (exceto $\mathrm{q}=90$ ) no final do período, indicando queda da desigualdade salarial entre os grupos de escolaridade. ${ }^{9}$

O cenário geral mostra, portanto, que a análise vista por quantis acentua a convergência entre os quantis e estratos educacionais, assim como um padrão de relativa constância para os quantis mais altos; enquanto a análise vista por estratos educacionais enfatiza a queda relativa dos rendimentos do ensino fundamental e a relativa constância dos rendimentos do estrato da 
pós-graduação. Os resultados apontam, em conjunto, que os rendimentos dos quantis mais altos e dos estratos educacionais de maiores anos de escolaridade (em especial a pós-graduação) seguem um comportamento distinto dos demais, sendo os que menos exibiram mudanças durante o período. Esses resultados, para o Brasil, se afiliam aos de Buchinsky (1994 e 1995), na medida em que também aqui se detectam retornos maiores à escolaridade para os indivíduos que se situam no topo da distribuição de salários. O trabalho de Martins e Pereira (2004) sublinha algumas explicações para resultados similares aos encontrados para o mercado de trabalho brasileiro. No trabalho dos autores para 16 países, o resultado mais robusto encontrado é de que os diferenciais de escolaridade têm impacto positivo sobre as desigualdades salariais, espalhando os aumentos dos retornos para os quantis de escolaridade maiores. As explicações plausíveis encontradas pelos autores é a existência de over-education (quando os indivíduos com maior escolaridade adquirem empregos que exigem menor qualificação), ou a possibilidade de haver interação entre escolaridade e habilidade, na qual o mais capaz pode se beneficiar de sua escolaridade e pagar por treinamento adicional, aumentando a distância entre o mais e o menos capaz para níveis educacionais maiores.

No gráfico 3 pode-se visualizar a diferença em pontos percentuais (eixo vertical) das variáveis categóricas de cada estrato de escolaridade para cada quantil. A ideia é mostrar, na diferença entre o ano de início (1996) e o ano de término (2004), quem ganhou e quem perdeu em termos de rendimento relativo. $\mathrm{O}$ resultado para o $10^{\circ}$ e para o $25^{\circ}$ quantis é que, nesse período, o estrato com ensino fundamental I foi o único com sinal positivo, sendo o estrato de pós-graduação o que mais perdeu. No $50^{\circ}$ e $75^{\circ}$ quantis, entretanto, todos os estratos escolares experimentaram perdas relativas (lembrando que o grupo de base para as dummies são indivíduos sem escolaridade); o estrato de pós-graduação deteve menores perdas; o estrato de ensino fundamental I teve os sinais negativos mais significantes. Para o quantil $90^{\circ}$, o comportamento dos coeficientes é semelhante, embora aqui o maior sinal negativo seja para o estrato do ensino fundamental.

As variáveis que não se relacionam diretamente com o estudo da posição relativa dos diversos grupos de escolaridade aos rendimentos são usadas para controlar efeitos dos indivíduos nos ganhos que não são contemplados 
Gráfico 3: Diferença de pontos percentuais das variáveis de rendimento por escolaridade entre os anos 1996 e 2004 para os diversos quantis e estratos de educação

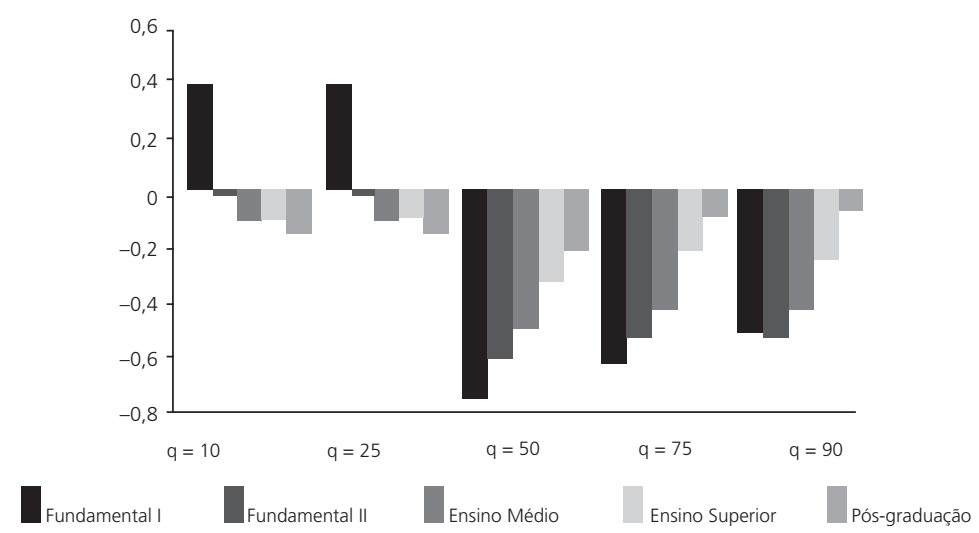

Fonte: Construção a partir das variáveis categóricas das regressões. Todas as variáveis têm significância inferior a 5\%.

unicamente pelo investimento em educação. Foram incluídas nas estimativas como variáveis de controle, não fazendo parte do foco principal do estudo. Entretanto, a análise da situação formal com o uso das regressões quantílicas traz resultados para os diversos pontos da distribuição, que tornam importante algum comentário a seu respeito. Tais resultados encontram-se expostos no gráfico 4.

Percebe-se, com o gráfico 4, que o atributo de carteira assinada é negativamente relacionado com o quantil da distribuição em que se insere o trabalhador. O eixo vertical mostra a diferença percentual dos rendimentos dos trabalhadores formais com relação aos informais, tudo o mais mantido constante. Visualiza-se que, quanto mais alto na distribuição quantílica de salários, menor a importância da diferença a favor dos trabalhadores formais. Para o quantil superior $90^{\circ}$, a situação de emprego formal parece influir negativamente nos rendimentos com relação à situação informal. De fato, é reconhecido na literatura que os empregadores tendem a registrar em carteira um salário menor que o efetivamente recebido para trabalhadores estabelecidos nos quantis superiores da distribuição. Uma possível explicação para esse resultado é que eles recebem um prêmio compensatório em salários pela ausência dos benefícios garantidos pela carteira assinada. Para motivar a permanência dos trabalhadores informais nessa situação, é possível que estes recebam um "prêmio salarial" tal que, dada sua escolaridade, detenham seus 

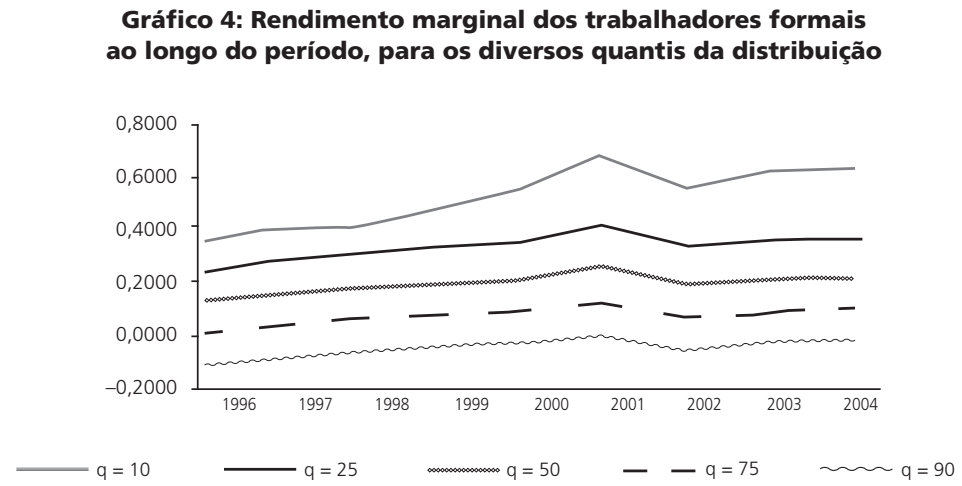

Fonte: Construção a partir das variáveis categóricas das regressões. Todas as variáveis têm significância inferior a 5\%.

rendimentos na posição superior da distribuição. Além disso, pode-se argumentar que é institucionalmente comum que o atributo de carteira assinada deixe de ser um atributo relativamente importante para algumas categorias de profissionais liberais ou freelancers, dadas as peculiaridades de suas atividades, ${ }^{10}$ o que faria com que essas categorias predominassem, controlada a escolaridade, no extremo superior da distribuição dos salários.

Para todos os quantis houve uma evolução positiva dos rendimentos dos trabalhadores formais em relação aos informais, atingindo pico em 2001. Para os quantis inferiores, a participação no mercado formal de trabalho garante substanciais acréscimos de rendimento. No trabalho de Menezes Filho et al. (2004), são estimadas equações de salário como função da situação de formalidade utilizando-se pseudopainéis e uma metodologia que controla o viés de autosseleção na escolha do setor. Os autores encontraram que, condicional ao nível de escolaridade, a remuneração do setor informal supera a do setor formal; a proteção legal não garantiria acréscimos salariais aos indivíduos. As vantagens oferecidas pelo setor formal seriam compensadas no setor informal por uma remuneração adicional. Os autores apontam, ainda, que a existência de viés de autosseleção no mercado de trabalho indica que, condicional ao nível de escolaridade, o diferencial de salários observado entre formais e informais decorre da melhor qualidade da força de trabalho no setor formal. Pode haver convergência desses resultados com as conclusões derivadas aqui, mas apenas para o topo da distribuição dos salários, na medida em que nesse estrato os resultados para a dummy do setor formal aparecem com sinal negativo. 
É importante notar que este trabalho se absteve de acrescentar variáveis que caracterizassem a categoria de ocupação dos trabalhadores, por simplificação. ${ }^{11}$

\section{CONCLUSÕES}

Este trabalho investiga se existem modificações relevantes entre os quantis de rendimento nos retornos por escolaridade (ou seja, se os retornos por escolaridade mudaram ao longo do período para quantis diferentes) e entre estratos educacionais para os diversos quantis de rendimento (os rendimentos relativos por quantil mudaram entre indivíduos com anos de escolaridade formal diverso). Para isso, foram utilizadas regressões quantílicas e variáveis categóricas para cada estrato educacional, utilizando dados para o período de 1996 a 2004 a partir da Pnad. Foram derivadas variáveis categóricas para os diversos estratos educacionais como estratégia para acompanhar a evolução relativa dos rendimentos dessas categorias ao longo do período. Além disso, a técnica das regressões quantílicas revelou que o comportamento das variáveis ao longo da distribuição não foi o mesmo: trabalhadores formais no quantil inferior recebem mais, ceteris paribus, enquanto para os trabalhadores formais dos quantis superiores a posse de carteira assinada é um atributo negativo.

O cenário geral dos resultados mostra que a análise vista por quantis acentua a convergência entre os quantis e estratos educacionais e a perda relativa do ensino superior ao longo do período, assim como um padrão de relativa constância para os quantis mais altos; enquanto a análise vista por estratos educacionais enfatiza a queda relativa dos rendimentos do ensino fundamental e a relativa constância dos rendimentos do estrato da pós-graduação. Os resultados apontam, em conjunto, que os rendimentos dos quantis mais altos e dos estratos educacionais de maiores anos de escolaridade seguem um comportamento distinto dos demais, sendo os que menos exibiram mudanças durante o período. Esses resultados, para o Brasil, se afiliam aos de Buchinsky (1994 e 1995), na medida em que também aqui se detectam retornos maiores à escolaridade para os indivíduos que se situam no topo da distribuição de salários.

Em uma breve análise nas características individuais dos trabalhadores, constatou-se que, quanto mais alto na distribuição quantílica de salários 
está o indivíduo, menor a importância da diferença a favor dos trabalhadores formais. Para o quantil superior $90^{\circ}$, a situação de emprego formal parece influir negativamente nos rendimentos com relação à situação informal.

A análise da conformação da oferta dos indivíduos em face de seus anos de escolaridade mostra significativo aumento da inserção relativa de trabalhadores mais escolarizados no mercado de trabalho urbano. Entretanto, uma análise detalhada da influência dos setores em que cada indivíduo se encontra empregado, como subsídio adicional às regressões, fica como uma possível extensão do trabalho no futuro.

\section{NOTAS}

1. Para Barros e Mendonça (2000), o estudo dos diferenciais salariais por nível de escolaridade tem outras finalidades, entre as quais três podem ser destacadas: (i) revela o montante adicional em salário resultado de maior escolaridade, ou o tamanho do incentivo para os indivíduos adquirirem mais educação; (ii) sendo os salários proporcionais à produtividade do trabalhador, e dados os níveis de escolaridade, os diferenciais de salários são uma proxy do impacto da educação sobre a produtividade e, através dos canais conhecidos, sobre o crescimento econômico; (iii) os diferenciais de salários informam como as desigualdades educacionais determinam desigualdades salariais.

2. Dentro do período excetua-se o ano 2000, ano em que a pesquisa da Pnad não foi realizada.

3. Existem explicações alternativas, como a de Spence (1973), que salienta que a educação age, na verdade, como um filtro ou como sinalização de produtividade durante a seleção pelo empregador. Arrow (1973) levanta a hipótese de seleção argumentando que a produtividade dos trabalhadores é desconhecida pelos empregadores no momento da contratação. Os empregadores utilizam a escolaridade dos empregados como uma proxy de sua produtividade potencial. Nos setores competitivos, a produtividade é valorizada e os retornos salariais à educação serão mais elevados. Nos setores menos competitivos, os retornos à educação depois da contratação serão menores.

4. É suposto que seis anos é a idade em que o indivíduo começa a estudar.

5. Para detalhes, consultar Mincer (1974), Becker (1975) e a explicação didática de Ehrenberg e Smith (2000).

6. Esses são os pesos das unidades amostrais (aqueles fornecidos nos arquivos de microdados), os quais foram incorporados na análise através das opções de ponderação disponíveis no software estatístico Stata 9.0. Essa ponderação é feita pelo procedimento analytical weights. A não utilização desses pesos implicaria estimativas tendenciosas.

7. Como as dummies foram construídas tendo como base os indivíduos sem escolaridade, alterações no grupo-base podem ser a causa de alterações nas relações entre as dummies 
estudadas aqui. A hipótese é de que esse grupo manteve seu comportamento com relação aos retornos por escolaridade constante o suficiente para permitir a análise. Ainda que tenha havido flutuação de comportamento para o grupo-base, supõe-se que de forma aproximada ela tenha se refletido igualmente para todos os estratos educacionais, de forma a pouco afetar a análise relativa entre os grupos estudados.

8. Os coeficientes devem ser multiplicados por 100 para serem interpretados como relações de porcentagem.

9. A explicação desse comportamento pode ser bem ampla. Como aponta Arbache (1999), a convergência dos salários e a grande competitividade no mercado de trabalho atualmente observadas não devem ser exclusivamente relacionadas a um só motivo, como, por exemplo, a abertura comercial e as reformas com orientação pró-mercado do período após a era Collor. Outros fatores, como a estabilização da inflação, a inserção do investimento estrangeiro direto, a consolidação da estabilidade política, a redução na força de atuação dos sindicatos, o aumento real do salário mínimo, entre outros, podem ter contribuído para os resultados evidenciados no gráfico 2 .

10. Maior flexibilidade de horários e atendimentos a clientes, múltiplas jornadas permitidas pela situação informal etc.

11. Entretanto, como comenta Arbache (1999), enquanto as variáveis de capital humano ainda se mostram como os principais fatores determinantes dos salários durante a década de 1990, a afiliação industrial, um fator tradicional determinante de segmentação de salários (em especial durante a década de 1980), teve sua importância decrescida ao longo dos anos 1990. Em 1996, a afiliação industrial estava em sétimo lugar em importância na determinação dos diferenciais de rendimentos, atrás de todas as variáveis que relacionavam capital humano, ocupação, assim como outras variáveis categóricas, tais como o diferencial por gênero e o pagamento de hora extra.

\section{REFERÊNCIAS BIBLIOGRÁFICAS}

ARBACHE, J. S. How do economic changes affect the structure of wages: the case of Brazilian manufacturing, 1984-1996. In: ROYAL ECONOMIC SOCIETY CONFERENCE, 1999, Nottingham, 1999.

ARROW, K. Higher education as a filter. Journal of Public Economics, n. 2, p. 193-216, 1973.

BARROS, R. P.; MENDONÇA, R. Salário e Educação no Brasil. Projeto Nordeste/Fundescola, 2000. (Série Estudos, n. 10.)

; FOGUEL, M. O impacto da abertura comercial sobre o mercado de trabalho brasileiro. Águas de Lindoia: Brazilian Economic Meeting, 24, 1996. Annals.

BECKER. G. Human Capital: a theoretical and empirical analysis, with special reference to education. Chicago: University of Chicago Press, 1975.

BUCHINSKY, M. Changes in the US wage structure, 1963-1987: application of quantile regression. Econometrica, n. 62, p. 405-458, 1994. 
Quantile regression, Box-Cox transformation model, and the U. S. wage structure, 1963-1987. Journal of Econometrics, v. 65, n. 1, p. 109-154, jan. 1995.

Recent advances in quantile regression models: a pratical guideline for empirical research. Journal of Human Resources, v. 33, n. 1, p. 88-126, 1998.

CHAMBERLAIN, G. Quantile regression, censoring and the structure of wages. In: SIMS, C. (Eds.). Advances in Econometrics 6th World Congress, Cambridge, v. 1, 1994.

DAVIS, S. J.; HALTIWANGER, J. Gross job creation, gross job destruction, and employment reallocation. The Quarterly Journal of Economics, v. 107, n. 3, p. 819-863, 1992.

EHRENBERG, R.; SMITH, R. A moderna economia do trabalho: teoria e politica pública. 5. ed. [S. 1.]: Makron Books, 2000.

FITZENBERGER, B.; KURZ, C. New insights on earnings trends across skill groups and industries in west gesmany. Universirat Konstantz, 1997. (mimeo.)

HARTOG, J.; PEREIRA, P. T.; VIEIRA, J. A. C. Changing returns to education in Portugal during the 1980s and early 1990s: OLS and quantile regression estimators. Applied Economics, v. 33, n. 8, p. 1.021-1.037, 2001.

JUSTO, W. R. Capital humano diminui desigualdades? Evidências para o Brasil a partir de regressões quantílicas. In: ENCONTRO REGIONAL DA ABET, 6. Anais. João Pessoa, 2007.

KOENKER, R.; BASSET, G. Regression quantiles. Econometrica, n. 46, p. 33-50, 1978.

MACHADO J. A. F.; MATA J. Counterfactual decomposition of changes in wage distributions using quantile regression. Journal of Applied Econometrics, 2005.

MARTINS, P; PEREIRA, P. Does education reduce wage inequality? Quantile regression evidence from 16 countries. Labour Economics, 2004.

MENEZES FILHO, N.; MENDES, M., ALMEIDA, E. O diferencial de salários formal-informal no Brasil: segmentação ou viés de seleção? Revista Brasileira de Economia, v. 58, n. 2, 2004.

MENEZES FILHO, N. A.; FERNANDES, R.; PICCHETTI, P. Rising human capital but constant inequality: the education composition effect in Brazil. Revista Brasileira de Economia, v. 60, n. 4, p. 407-424, dez. 2006.

MINCER, J. Schooling, Experience, and Earnings. Nova York: NBER Press, 1974.

MITNER, C.; WRIGHT, P. Modelling labour market adjustment to trade liberalisation in an industrializing economy. Economic Journal, n. 108, 1998.

MOREIRA, M. M.; NAJBERG, S. Abertura comercial: criando ou exportando empregos? Texto para Discussão, Rio de Janeiro: Depec - Banco de Desenvolvimento Econômico e Social, n. 59, 1997.

PAUL, E. A.; ROBINSON, M. D. The implications of increasing economic openness for real wages in developing countries, 1973-1990. World Development, n. 25, 1997.

RAMOS, L. A Desigualdade de rendimentos do trabalho no período pós-real: o papel da escolaridade e do desemprego. Economia Aplicada, v. 11, n. 2, p. 281-301, 2007.

REVENGA, A. Employment and wage effects of trade liberalization: the case of mexican manufacturing. Journal of Labor Economics, n. 15, 1997. 
SALM, C.; SABOIA, J.; CARVALHO, P. G. M. Produtividade na indústria brasileira: questões Metodológicas e novas evidências empíricas. Pesquisa e Planejamento Econômico, n. 27, 1997.

SILVEIRA NETO, R. da M.; CAMPELO, A. K. O perfil das disparidades regionais de renda no Brasil: evidências a partir de regressões quantílicas para os anos de 1992 e 2001. ENCONTRO NaCional De ECONOMIA, 31. Anais. Porto Seguro, 2003.

SPENCE, M. Job market signaling. The Quarterly Journal of Economics, v. 87, n. 3, p. 355-374, 1973.

YU, K.; LU, Z.; STANDER, J. Quantile regression: applications and current research areas. The Statistician, v. 52, n. 3, p. 331-350, 2003. 